\title{
Retrospective Study of the Survival of Patients who Underwent Cardiopulmonary Resuscitation in an Intensive Care Unit
}

\author{
Daniel Martins Moreira, Guilherme Mariante Neto, Marcelo Wierzynski Oliveira, Letícia Biscaino Alves, \\ Luís Carlos Chorazje Adamatti, Eliana de Andrade Trotta, Sílvia Regina Rios Vieira \\ Porto Alegre, RS - Brazil
}

Objective - To evaluate clinical and evolutive characteristics of patients admitted in an intensive care unit after cardiopulmonary resuscitation, identifying prognostic survival factors.

Methods - A retrospective study of 136 patients admitted between 1995 and 1999 to an intensive care unit, evaluating clinical conditions, mechanisms and causes of cardiopulmonary arrest, and their relation to hospital mortality.

Results - A 76\% mortality rate independent of age and sex was observed. Asystole was the most frequent mechanism of death, and seen in isolation pulmonary arrest was the least frequent. Cardiac failure, need for mechanical ventilation, cirrhosis and previous stroke were clinically significant $(p<0.01)$ death factors.

Conclusion - Prognostic factors supplement the doctor's decision as to whether or not a patient will benefit from cardiopulmonary resuscitation.

Keywords: cardiopulmonary resuscitation, cardiopulmonary arrest, intensive care unit
Hospital de Clínicas de Porto Alegre da Faculdade de Medicina da UFRGS Mailing address: Daniel Martins Moreira - Rua Machado de Assis, 673 - 90620-260 Porto Alegre, RS - Brazil - E-mail: dam@ufrgs.br
Cardiopulmonary resuscitation is a therapeutic medical modality with both indications and counterindications ${ }^{1,2}$. Although frequently performed in the hospital, its efficacy has not been well established in this environment where many seriously ill patients are found who have multisystem afflictions and a poor prognosis. Inappropriate resuscitation measures may result in increased financial and emotional costs and little or no benefits to the patient.

Unsatisfactory results of cardiopulmonary resuscitation procedures have for the most part attributed to its indiscriminate use. Various individual factors interfere with recovery from cardiopulmonary arrest. Despite the fact that the prediction of an individual outcome of such resuscitation is of great medical, ethical, and socio-economic interest, doubts about the decision whether or not to resuscitate a given patient, as well as about the consequences of either attitude, persist ${ }^{3}$. This decision should not be made only at the moment of cardiopulmonary arrest, but should also take the previous medical condition of the patient into account ${ }^{4}$. Therefore, cardiopulmonary manouvers should be critically indicated for select patients, taking into consideration criteria that may be related to a better prognosis after therapy. The definition of these criteria is of relevance, rendering important knowledge about pre, trans and postarrest conditions of patients who undergo cardiopulmonary resuscitation.

It is the objective in the present work to evaluate clinical characteristics and evolution after cardiopulmonary resuscitation of patients confined to the intensive care unit of the Hospital de Clínicas de Porto Alegre. We identified factors that may influence their prognosis to a significant extent, thus stimulating reflection about the critical utilization of cardiopulmonary resuscitation.

\section{Methods}

The present work consists of a review of case histories of 136 patients admitted between January 1995 and Decem- 
ber 1999 to the intensive care unit of the Hospital de Clínicas de Porto Alegre after successfully undergoing cardiopulmonary resuscitation manouvers. The Ethics and Research Commission of the hospital approved this study.

Patients were surveyed for sex, age, and mechanisms, causes and recurrence of cardiopulmonary arrest, and clinical conditions before and after the event as well as the incidence of mortality. Mechanisms of cardiopulmonary arrest were classified as ventricular fibrillation, pulseless ventricular tachycardia, pulseless electrical activity, asystole, or respiratory arrest without cardiac arrest. Causes were divided into acute myocardial infarct, cardiac ischemia or arrhythmia, respiratory insufficiency, hydroelectrolyte disturbances, hypotension, and others. Clinical conditions like arterial pressure, presence of pneumonia, sepsis, cancer, angina pectoris, acute myocardial infarction, congestive heart failure, stroke, coma, oliguria, elevated serum urea, $(>100 \mathrm{mg} /$ dL) cirrhosis, use of mechanical ventilation and bedrest were evaluated.

Patients were evaluated regarding the major outcome, namely, hospital mortality. Secondary outcomes were mechanisms and causes of cardiorespiratory arrest. Statistical analysis was performed with the SPSS for Windows 10 program, using the Student $t$ test for quantitative and the chisquare test for qualitative data, multiple logistic regression for risk factors, and prognosis and residue analysis for the mechanism of cardiopulmonary arrest. The level of significance adopted was $\alpha=0.05$.

\section{Results}

The population's mean age was of 60 years, and gender distribution was homogeneous: 67 men and 69 women. General intrahospital mortality was $75.7 \%$ (103 patients) without a significant difference regarding gender. No significant difference existed between the mean age of groups who died or survived (tab. I). Presumed causes of cardiorespiratory arrest were acute myocardial infarction, cardiac ischemia, or arrhythmia in $27(20 \%)$, respiratory insufficiency in $27(20 \%)$, hypotension in $24(17 \%)$, and hydroelectrolyte or acid/base equilibrium disturbances in $10(7 \%)$ patients; other causes including pulmonary embolism, intracerebral hemorrhage, stroke, intoxication, pacemaker dysfunction, neoplasm infiltration of the central nervous system were observed in $21(15.4 \%)$, and unknown causes existed in 27 $(20 \%)$ patients. No significant differences regarding causes

\begin{tabular}{|c|c|c|c|c|}
\hline \multicolumn{5}{|c|}{$\begin{array}{l}\text { Table I - Profile of patients who underwent cardiopulmonary } \\
\text { resuscitation }\end{array}$} \\
\hline \multicolumn{2}{|c|}{ Characteristic } & Deaths & No deaths & $\mathrm{p}$ \\
\hline \multicolumn{2}{|c|}{ Age (years) } & $61 \pm 16$ & $57 \pm 18$ & 0.31 \\
\hline \multirow[t]{2}{*}{ Gender } & Male & $52(77.3 \%)$ & $15(22.7 \%)$ & \\
\hline & Female & $51(73.9 \%)$ & $18(26.1 \%)$ & 0.69 \\
\hline
\end{tabular}

of cardiorespiratory arrest were observed between survivors and nonsurvivors (tab. II).

The predominant mechanisms responsible for cardiorespiratory arrest were ventricular fibrillation and pulseless ventricular tachycardia, observed in 25 patients totalling $18.3 \%$ ( $13.1 \%$ and $5.1 \%$, respectively). Asystole was observed in $21(14.4 \%)$, respiratory arrest alone in $11.8 \%$, and pulseless electrical activity in $11.1 \%$ of the patients. Based on a review of case histories, it was impossible to determine the mechanism of cardiopulmonary arrest in 59 (43.4\%) patients. Upon comparison regarding the mechanism of cardiopulmonary arrest between survivors and nonsurvivors, a significantly higher mortality was observed in the asystole group $(\mathrm{P}=0.02)$ and a lower mortality $(\mathrm{P}=0.001)$ in the group with respiratory but not cardiac arrest. No significant differences existed between general mortality and groups exhibiting ventricular fibrillation, ventricular tachycardia, and pulseless electrical activity (tab. III). Among death risk factors of patients who underwent cardiopulmonary resuscitation, congestive heart failure, need for mechanical ventilation, recent stroke and hepatic cirrhosis increased such risk significantly (tab. IV). The recurrence of cardiopulmonary arrest that occurred in $27(19.9 \%)$ patients was also strongly associated with a worse prognosis $(\mathrm{R} R=1.4 ; 95 \%$ $\mathrm{CI}=1.18-1.57$ ).

\begin{tabular}{|c|c|c|c|c|c|}
\hline \multicolumn{6}{|c|}{ Table II - Cause of cardiopulmonary arrest } \\
\hline \multirow[t]{2}{*}{ Characteristic } & \multicolumn{2}{|c|}{ Deaths } & \multicolumn{2}{|c|}{ No deaths } & \multirow[t]{2}{*}{$\mathrm{p}$} \\
\hline & $\mathrm{N}^{\mathrm{o}}$ & $\%$ & $\mathrm{~N}^{\mathrm{o}}$ & $\%$ & \\
\hline \multicolumn{6}{|l|}{ Hydroeletrolyte and/or } \\
\hline $\begin{array}{l}\text { AMI, arrhythmia, or } \\
\text { myocardial ischemia }\end{array}$ & 20 & $74.1 \%$ & 7 & $25.9 \%$ & 0.97 \\
\hline Hypotension & 18 & $75 \%$ & 6 & $25 \%$ & 0.86 \\
\hline Respiratory insufficiency & 19 & $70.4 \%$ & 8 & $29.6 \%$ & 0.63 \\
\hline Others ${ }^{1}$ & 18 & $85.7 \%$ & 3 & $14.3 \%$ & 0.38 \\
\hline Ignored & 19 & $70.4 \%$ & 8 & $29.6 \%$ & 0.63 \\
\hline Total & 103 & $75.7 \%$ & 33 & $24.3 \%$ & - \\
\hline \multicolumn{6}{|c|}{$\begin{array}{l}\text { AMI- acute myocardial infarct; others }{ }^{1} \text { - pulmonary embolism, } \\
\text { intracerebral hemorrhage, ischemic vascular cerebral accident, } \\
\text { intoxication, pacemaker dysfunction, neoplasm infiltration in the cen- } \\
\text { tral nervous system. }\end{array}$} \\
\hline
\end{tabular}

\begin{tabular}{|lccccc|}
\hline \multicolumn{5}{|c|}{ Table III - Mechanism of cardiorespiratory arrest } \\
\hline Characteristic & \multicolumn{3}{c|}{ Deaths } & No deaths & $\mathrm{p}$ \\
& $\mathrm{N}^{\mathrm{o}}$ & $\%$ & $\mathrm{~N}^{\mathrm{o}}$ & $\%$ & \\
\hline \multirow{2}{*}{ VF and pulseless VT } & 17 & $68 \%$ & 8 & $32 \%$ & 0.61 \\
Ventricular fibrillation & 13 & $72.2 \%$ & 5 & $27.8 \%$ & 0.92 \\
Pulseless VT & 4 & $57.1 \%$ & 3 & $42.9 \%$ & 0.37 \\
Asystole & 20 & $95.2 \%$ & 1 & $4.8 \%$ & 0.02 \\
Pulseless electrical activity & 11 & $73.3 \%$ & 4 & $27.7 \%$ & 0.84 \\
Respiratory arrest only & 7 & $43.8 \%$ & 9 & $56.2 \%$ & 0.001 \\
Ignored & 48 & $81.4 \%$ & 11 & $18.6 \%$ & 0.19 \\
Total & 103 & $75.7 \%$ & 33 & $24.3 \%$ & - \\
\hline \multirow{2}{*}{ VF- ventricular fibrillation; VT- ventricular tachycardia. } \\
\hline
\end{tabular}




\begin{tabular}{|lcccccccc|}
\hline \multicolumn{8}{|c|}{ Table IV - Death risk factors in cardiopulmonary arrest patients } \\
\hline Factor & \multicolumn{2}{c}{ Deaths } & \multicolumn{2}{c|}{ No deaths } & RR & p & CI $95 \%$ \\
& $\mathrm{~N}^{\circ}$ & $\%$ & $\mathrm{~N}^{\circ}$ & $\%$ & & & \\
\hline SAP $<100 \mathrm{mmHg}$ & 34 & $33 \%$ & 8 & $24.2 \%$ & 1.07 & 0.52 & $0.85-1.35$ \\
Pneumonia & 22 & $21.4 \%$ & 6 & $18.2 \%$ & 1.04 & 0.71 & $0.84-1.28$ \\
Ureia $>100 \mathrm{mg} / \mathrm{dL}$ & 9 & $8.7 \%$ & 2 & $6.1 \%$ & 0.88 & 0.56 & $0.57-1.34$ \\
Cancer & 24 & $23.3 \%$ & 5 & $15.2 \%$ & 1.15 & 0.24 & $0.9-1.46$ \\
Coma & 14 & $13.6 \%$ & 2 & $6.1 \%$ & 0.95 & 0.7 & $0.73-1.23$ \\
Angina & 6 & $5.8 \%$ & 5 & $15.2 \%$ & 0.95 & 0.87 & $0.52-1.72$ \\
AMI & 7 & $6.8 \%$ & 6 & $18.2 \%$ & 0.66 & 0.11 & $0.4-1.1$ \\
CHF & 21 & $20.4 \%$ & 2 & $6.1 \%$ & 1.51 & $<0.001$ & $1.22-1.86$ \\
Oliguria & 12 & $11.7 \%$ & 4 & $12.1 \%$ & 0.89 & 0.51 & $0.63-1.25$ \\
Sepsis & 24 & $23.3 \%$ & 4 & $12.1 \%$ & 1.06 & 0.54 & $0.87-1.28$ \\
MV & 17 & $16.5 \%$ & 0 & $0 \%$ & 1.38 & $<0.001$ & $1.15-1.66$ \\
Stroke & 15 & $14.6 \%$ & 0 & $0 \%$ & 1.28 & 0.003 & $1.08-1.52$ \\
Cirrhosis & 6 & $5.8 \%$ & 0 & $0 \%$ & 1.55 & $<0.001$ & $1.23-1.95$ \\
\hline
\end{tabular}

RR - risk relative; SAP - systolic arterial pressure; AMI - acute myocardial infarction; $\mathrm{CHF}$ - congestive heart failure; $\mathrm{MV}$ - mechanical ventilation.

\section{Discussion}

Cardiopulmonary resuscitation is frequently performed in stressful situations. Persons providing this treatment frequently fail to consider the possibility that the patient may have a cardiac disease in its final phase, taking into account only that cardiopulmonary arrest is an emergency situation. Roberts et al ${ }^{5}$ concluded that doctors and nurses expect cardiopulmonary arrest patients to have a survival rate higher than they really do. In their study, doctors were shown to expect $24 \%$ of cardiopulmonary resuscitation procedures in adults and $41 \%$ in children to be efficient, while nurses expected survival indexes of $30 \%$ and $45 \%$ for adults and children, respectively. Laymen had an even higher level of expectation: a 52\% cardiopulmonary arrest reversion rate was expected for adults and a $63 \%$ reversion rate for children. Several studies have shown hospital discharge rates following cardiopulmonary resuscitation to vary between 11 and $39 \%$. In the present study, a $24.3 \%$ discharge ratio was observed.

Possible mortality risk factors reinforce a doctor's decision as to whether or not the patient will benefit from cardiopulmonary resuscitation. In agreement with the literature ${ }^{3,7,8}$, the present study found the following statistically significant mortality risk factors: congestive heart failure, need for mechanical ventilation, previous stroke and cirrhosis. Some risk factors appearing as statistically significant in the literature, such as sepsis ${ }^{7}$ and hypotension ${ }^{3,9}$, were not characterized in the present study. Patient age is a contro- versial risk factor. No significant difference between the average age of the patients who died in the hospital and those who were discharged was found in this work. In agreement with the literature, previous data suggest that sex does not alter death rates after cardiopulmonary arrest ${ }^{4}$. Some risk factors described in the literature, such as the Glasgow coma scale, acute organ failure ${ }^{10}$, diabetes mellitus ${ }^{3}$, physiological scores including APACHE $2^{4,8}$, and duration of cardiopulmonary resuscitation ${ }^{8,11,12}$, were not studied in the present work.

No cause with a statistically significant relation to mortality or patient survival was found (tab. II). These results differ from some data in the literature showing hypotension and acute myocardial infarction as causes of a worsened prognosis of cardiopulmonary arrest ${ }^{9}$. Further work will have to be performed to better evaluate these factors' influence on the prognosis of cardiopulmonary arrest patients.

It is important to point out the relationship between mortality and the mechanism of cardiorespiratory arrest. Only 2 statistically significant mechanisms were found: asystole associated with the worst prognosis and respiratory arrest without cardiac arrest associated with the best prognosis. Other studies had shown mortality reduction for ventricular fibrillation and tachycardia ${ }^{9,11-13}$, results not confirmed in the present study. This difference may have been due to the smaller number of patients having their cardiopulmonary arrest mechanism explained on the basis of the retrospective analysis of their case histories ( $57 \%$ of 136 patients).

Despite the great importance of patient prognosis, it must be mentioned that the decision whether to resuscitate cardiopulmonary arrest victims or not rests with the physician responsible for the case. He in turn may use literature data to aid him in the decision of whether or not to perform cardiopulmonary resuscitation manouvers. The prognostic scores studied do not substitute medical decision. In case of doubts about the most correct decision, the doctor should opt for cardiopulmonary resuscitation.

\section{Acknowlegements}

To Drs. Fernando Procianoy, Jorge Guardiola Meinhardt Júnior, Diego da Fonseca Mossmann, Renan Desimon Cabral, Diego Chemello, Thiago Quedi Furian, Fabiane Neiva Backes, Leandro de Moura, Alexsandro de Lucena Theil, Cristiane Paim Rigol, for collaboration rendered. 


\section{References}

1. Prado KF, Loss SH, Bohel JA, Vieira SRR. Estudo de 87 pacientes internados no CTI após reanimação cardiopulmonar. Rev Bras Terap Intens 1990; 2: 90-4.

2. The American Heart Association in collaboration with The International Liaison Committee on Resuscitation (ILCOR). Guidelines 2000 for Cardiopulmonary Resuscitation and Emergency Cardiovascular Care. Circulation 2000; 102(suppl I): I1-384.

3. Rogove HJ, Safar P, Sutton Tyrrell K, Abramson NS. Old age does not negate good cerebral outcome after cardiopulmonary resuscitation: analyses from the brain resuscitation clinical trials. The Brain Resuscitation Clinical Trial I and II Study Groups. Crit Care Med 1995; 23: 18-25.

4. Landry FJ, Parker JM, Phillips YY. Outcome of cardiopulmonary resuscitation in the intensive care setting. Arch Intern Med 1992; 152: 2305-8.

5. Roberts D, Hirschman D, Scheltema K. Adult and pediatric CPR: attitudes and expectations of health professionals and laypersons. Am J Emerg Med 2000, 18 : 465-8.

6. Araújo R, Gomes E, Lopes M, Araújo MS. Outcome of cardiopulmonary resuscitation in a Portuguese university hospital. Eur J Emerg Med 1997; 4: 81-6.

7. Staudinger T, Stoiser B, Müllner M, et al. Outcome and prognostic factors in criti- cally ill cancer patients admitted to the intensive care unit. Crit Care Med 2000; 28: 1322-8.

8. Peterson MW, Geist LJ, Schwartz DA, Konicek S, Moseley PL. Outcome after cardiopulmonary resuscitation in a medical intensive care unit. Chest 1991; 100: 168-74.

9. Di Bari M, Chiarlone M, Fumagalli S, et al. Cardiopulmonary resuscitation of older, inhospital patients: immediate efficacy and long-term outcome. Crit Care Med 2000; 28: 2320-5.

10. Smith DL, Kim K, Cairns BA, Fakhry SM, Meyer AA. Prospective analysis of outcome after cardiopulmonary resuscitation in critically ill surgical patients. J Am Coll Surg 1995; 180: 394-401.

11. Rajaram R, Rajagopalan RE, Pai M, Mahendran S. Survival after cardiopulmonary resuscitation in an urban Indian hospital. Natl Med J India 1999; 12: 51-5.

12. van Walraven C, Forster AJ, Stiell IG. Derivation of a clinical decision rule for the discontinuation of in-hospital cardiac arrest resuscitations. Arch Intern Med 1999; 159: 129-34.

13. Kuisma M, Määttä T, Repo J. Cardiac arrests witnessed by EMS personnel in a multitiered system: epidemiology and outcome. Am J Emerg Med 1998; 16: 12-6. 